\title{
Resonant Dimer Relaxation in Cold Atoms with a Large Scattering Length
}

\author{
Eric Braaten \\ Department of Physics, The Ohio State University, Columbus, OH 43210, USA \\ H.-W. Hammer \\ Helmholtz-Institut für Strahlen- und Kernphysik (Theorie), Universität Bonn, 53115 Bonn, Germany
}

(Dated: January 29, 2007)

\begin{abstract}
Efimov physics refers to universal phenomena associated with a discrete scaling symmetry in the 3-body problem with a large scattering length. The first experimental evidence for Efimov physics was the recent observation of a resonant peak in the 3 -body recombination rate for ${ }^{133} \mathrm{Cs}$ atoms with large negative scattering length. There can also be resonant peaks in the atom-dimer relaxation rate for large positive scattering length. We calculate the atom-dimer relaxation rate as a function of temperature and show how measurements of the relaxation rate can be used to determine accurately the parameters that govern Efimov physics.
\end{abstract}

PACS numbers: 21.45.+v,34.50.-s

Keywords: Few-body systems, scattering of atoms and molecules.

The Efimov effect is a remarkable phenomenon that can occur in a 3-body system of nonrelativistic particles with short-range interactions when the S-wave scattering length $a$ is tuned to $\pm \infty$. Vitaly Efimov discovered in 1970 that in this limit there can be infinitely many 3body bound states with an accumulation point at the scattering threshold and a geometric spectrum [1]: each successive bound state is shallower than the previous one by a multiplicative factor. In the case of identical bosons with mass $m$, this factor is $e^{2 \pi / s_{0}} \approx 515$, where $s_{0} \approx$ 1.00624. The binding energies of the Efimov states for $a= \pm \infty$ can be expressed as

$$
E_{T}^{(n)}=\left(e^{2 \pi / s_{0}}\right)^{n_{*}-n} \hbar^{2} \kappa_{*}^{2} / m,
$$

where $\kappa_{*}$ is the binding wavenumber of the branch of Efimov states labeled by $n_{*}$. The geometric spectrum is a signature of a discrete scaling symmetry with discrete scaling factor $e^{\pi / s_{0}} \approx 22.7$.

The Efimov effect is just one example of the universal phenomena characterized by a discrete scaling symmetry that govern the 3-body system in the scaling limit in which the range of the interaction is negligible compared to $|a|$ 2, 3]. For a review of these phenomena, which we refer to as Efimov physics, see Ref. [4]. For simplicity, we focus on the case of identical bosons. We refer to the particles as atoms, their 2-body bound states as dimers, and their 3-body bound states as trimers. If the scattering length $a$ is large and positive, there is a shallow dimer with binding energy $E_{D}=\hbar^{2} /\left(m a^{2}\right)$. Efimov pointed out that there is an infinite sequence of negative values of $a$ for which there is an Efimov trimer at the 3-atom scattering threshold [2]: $a=\left(e^{\pi / s_{0}}\right)^{n} a_{*}^{\prime}$, where $a_{*}^{\prime} \approx-1.56 \kappa_{*}^{-1}[5]$. There is also an infinite sequence of positive values of $a$ for which there is an Efimov trimer at the atom-dimer scattering threshold [2]: $a=\left(e^{\pi / s_{0}}\right)^{n} a_{*}$, where $a_{*} \approx 0.0708 \kappa_{*}^{-1}[5]$. Another example of Efimov physics is an infinite sequence of positive values of $a$ for which the 3-body recombination rate into the shallow dimer vanishes [6, 7, 8]. The universal aspects of Efimov physics are determined by two parameters: the scattering length $a$ and the Efimov parameter $\kappa_{*}$.

The alkali atoms used in most cold atom experiments have many deeply-bound diatomic molecules (deep dimers). Efimov physics is modified by the existence of the deep dimers, because Efimov trimers can decay into an atom and a deep dimer. Deep dimers also provide inelastic scattering channels for three atoms or for an atom and a dimer. If there is an Efimov trimer near the scattering threshold, it can give a resonant enhancement of inelastic scattering processes. When $a \approx\left(e^{\pi / s_{0}}\right)^{n} a_{*}^{\prime}$, the resonant inelastic process is 3 -atom recombination into a deep dimer [7, 9]. When $a \approx\left(e^{\pi / s_{0}}\right)^{n} a_{*}$, the resonant inelastic process is dimer relaxation, in which the collision of an atom and a shallow dimer produces an atom and a deep dimer [10]. If there are deep dimers, the universal phenomena associated with Efimov physics are determined by three parameters: $a, \kappa_{*}$, and a parameter $\eta_{*}$ that determines the widths of Efimov trimers [10].

Finally, more than 35 years after Efimov's discovery, the first experimental evidence for Efimov physics has emerged. In a beautiful experiment with cold ${ }^{133} \mathrm{Cs}$ atoms, the Innsbruck group has observed a resonance in the 3-body recombination rate that can be attributed to an Efimov trimer near the 3-atom threshold 11. They used the magnetic field to control the scattering length of ${ }^{133} \mathrm{Cs}$ atoms in the lowest hyperfine state by exploiting a Feshbach resonance at -12 G. Since inelastic 2body losses are energetically forbidden, the dominant loss mechanism is 3-body recombination. By varying the magnetic field from 0 to $150 \mathrm{G}$, they were able to change the scattering length from $-2500 a_{0}$ through 0 to $+1600 a_{0}$, where $a_{0}$ is the Bohr radius. They observed a giant loss feature at $a \approx-850 a_{0}$. At the lowest temperature at which they measured the loss rate as a function 
of $a$, which was $10 \mathrm{nK}$, it can be fit rather well by the universal formula of Refs. [4, 10] with $\eta_{*}=0.06(1)$.

The Innsbruck group also measured the 3-body recombination rate for positive values of $a$ reached by increasing the magnetic field through the zero of the scattering length 11]. They observed a local minimum in the inelastic loss rate at $a \approx 210 a_{0}$. The minimum could be due to Efimov physics, although the value of $a$ is not large compared to the van der Waals length scale for Cs atoms: $\left(m C_{6} / \hbar^{2}\right)^{1 / 4} \approx 200 a_{0}$. Another complication was that the lowest temperature that was reached for positive $a$ was $200 \mathrm{nK}$. Thermal effects can be taken into account if the 3-body recombination rate is known as a function of the collision energy. The universal predictions for nonzero energy have not yet been calculated.

One way to confirm the discovery of Efimov physics would be to observe a second loss feature related to the first feature by the discrete scaling symmetry. In the case of identical bosons, the large size of the discrete scaling factor $e^{\pi / s_{0}} \approx 22.7$ makes this difficult. Since the 3 body recombination rate scales like $a^{4}$, the loss rate at the second loss feature is larger than that at the first feature by a factor of $22.7^{4} \approx 2 \times 10^{5}$. Another way is to observe the correlations between different aspects of Efimov physics that are predicted by universality. This requires the accurate determination of the parameters $\kappa_{*}$ and $\eta_{*}$ that govern Efimov physics. It may also require the calculation of the universal predictions at nonzero temperature to account properly for thermal effects. The effects of temperature on the resonant inelastic loss for negative $a$ have been considered in Refs. [12, 13, 14].

For positive values of $a$, the Innsbruck group has also created mixtures of ${ }^{133} \mathrm{Cs}$ atoms and shallow dimers [15], which has allowed them to study atom-dimer collisions. The most dramatic manifestation of Efimov physics for $a>0$ is the resonant enhancement of dimer relaxation for $a$ near $\left(e^{\pi / s_{0}}\right)^{n} a_{*}$. The Innsbruck group has observed a resonance in inelastic atom-dimer collisions near $a \approx 400 a_{0}$ 15. In this paper, we calculate the rate for inelastic atom-dimer collisions in a cold gas of atoms and dimers as a function of the scattering length and the temperature. By measuring the position and line shape of the resonance and using our results to take into account the temperature, it should be possible to determine the Efimov parameters $\kappa_{*}$ and $\eta_{*}$ accurately. Once these parameters are determined, they can be used to make accurate predictions for other universal phenomena associated with Efimov physics.

The collision energy for an atom and a shallow dimer is $E=3 \hbar^{2} k^{2} /(4 m)$, where $k$ is the wavenumber of the atom or dimer in the center-of-mass frame. The differential cross section for elastic atom-dimer scattering is

$$
\frac{d \sigma_{A D}}{d \Omega}=\left|f_{A D}(k, \theta)\right|^{2},
$$

where $f_{A D}(k, \theta)$ is the scattering amplitude, which has the partial wave expansion

$$
f_{A D}(k, \theta)=\sum_{L=0}^{\infty} \frac{2 L+1}{k \cot \delta_{L}^{A D}(k)-i k} P_{L}(\cos \theta) .
$$

The elastic cross section $\sigma_{A D}(E)$ is obtained by integrating over the solid angle of $4 \pi$. The leading terms in the expansion of $k \cot \delta_{0}^{A D}(k)$ in powers of $k$ define the atomdimer scattering length $a_{A D}$ and the atom-dimer effective range $r_{s, A D}$ :

$$
k \cot \delta_{0}^{A D}(k)=-1 / a_{A D}+\frac{1}{2} r_{s, A D} k^{2}+\ldots .
$$

The optical theorem relates the total cross section, which is the sum of the elastic and inelastic cross sections, to the $\theta \rightarrow 0$ limit of the elastic scattering amplitude in Eq. (3):

$$
\sigma_{A D}^{(\text {total })}(E)=\frac{4 \pi}{k} \operatorname{Im} f_{A D}(k, \theta=0) .
$$

If there are no deep dimers, the scattering is elastic up to the dimer-breakup threshold at $E=E_{D}$.

We first consider the case in which there are no deep dimers, so the phase shifts $\delta_{L}^{A D}(k)$ are real-valued below the dimer-breakup threshold. The phase shifts for $L \geq 1$ are universal functions of $k a$ only. The $L=0$ phase shift is sensitive to 3-body interactions at short distances and therefore depends also on $a \kappa_{*}$. Efimov used unitarity to derive powerful constraints on the dependence on $a \kappa_{*}$ that he referred to as the radial law. He used the radial law to deduce an analytic expression for the atom-dimer scattering length $a_{A D}$ [3] up to numerical constants that were first calculated by Simenog and Sinitchenko [16]. They have been calculated more accurately using an effective field theory [5, 17, 18]. This method was used in Ref. [5] to calculate the atom-dimer effective range $r_{s, A D}$. These universal results are

$$
\begin{aligned}
a_{A D} & =\left(1.46+2.15 \cot \left[s_{0} \ln \left(a / a_{*}\right)\right]\right) a, \\
r_{s, A D} & =\left(1.13+0.73 \cot \left[s_{0} \ln \left(a / a_{*}\right)+0.98\right]\right)^{2} a .
\end{aligned}
$$

Numerical calculations indicate that $r_{s, A D}$ vanishes within the numerical accuracy when $a \approx\left(e^{\pi / s_{0}}\right)^{n} 4.85 a_{*}$ [5]. The above expression for $r_{s, A D}$ is compatible with Efimov's radial law and also satisfies this additional constraint.

We now consider the case in which there are deep dimers, so dimer relaxation provides inelastic atom-dimer scattering channels. The large binding energy of the deep dimer is released through the large kinetic energies of the recoiling atom and dimer. The event rate $\beta$ for dimer relaxation is defined so that the number of dimer relaxation events per time and per volume in a gas of atoms with number density $n_{A}$ and dimers with number density $n_{D}$ is $\beta n_{A} n_{D}$. If the atom and the deep dimer from the relaxation process have such large kinetic energies that 
they escape from the system, the rate of decrease in the number densities is

$$
\frac{d}{d t} n_{A}=\frac{d}{d t} n_{D}=-\beta n_{A} n_{D} .
$$

The event rate $\beta$ can be expressed in terms of a statistical average of the inelastic atom-dimer cross section:

$$
\beta=\frac{3 \hbar}{2 m}\left\langle k \sigma_{A D}^{\text {(inelastic) }}(E)\right\rangle .
$$

The inelastic cross section is the difference between the total cross section in Eq. (5) and the elastic cross section obtained by integrating Eq. (2) over angles. If the temperature is large compared to the critical temperature for Bose-Einstein condensation, the thermal average indicated by the angular brackets in Eq. (8) can be carried out using Boltzmann statistics. In a gas consisting of atoms and dimers in thermal equilibrium (but not chemical equilibrium), the Boltzmann factor is $\exp \left(-3 \hbar^{2} k^{2} /\left(4 m k_{B} T\right)\right)$.

In Ref. [10], we pointed out that in the scaling (or zerorange) limit, the cumulative effect of all the deep dimers on Efimov physics can be taken into account rigorously through one additional parameter $\eta_{*}$. The effects of Efimov physics can be dramatic only if $\eta_{*}$ is much less than 1. If the universal expression for a scattering amplitude for the case of no deep dimers is known as an analytic function of $\kappa_{*}$ or $a_{*}$, the corresponding result for a system with deep dimers can be obtained without any additional calculation simply by making the substitution

$$
\ln a_{*} \longrightarrow \ln a_{*}-i \eta_{*} / s_{0} .
$$

Since the $L=0$ atom-dimer phase shift is the only one that depends on $\kappa_{*}$, the $L=0$ channel is the only one that contributes to dimer relaxation at leading order. Making the substitution in Eq. (9) in Eqs. (6), we obtain complex-valued expressions for the atom-dimer scattering length and effective range:

$$
\begin{aligned}
a_{A D} & =\left(1.46+2.15 \cot \left[s_{0} \ln \left(a / a_{*}\right)+i \eta_{*}\right]\right) a, \quad(10) \\
r_{s, A D} & =\left(1.13+0.73 \cot \left[s_{0} \ln \left(a / a_{*}\right)+0.98+i \eta_{*}\right]\right)^{2} a .
\end{aligned}
$$

If the expansion for $k \cot \delta_{0}^{A D}(k)$ in Eq. (44) is truncated after the effective range term, the inelastic cross section reduces to

$$
\sigma_{A D}^{\text {(inelastic) }}(E)=\frac{4 \pi\left(-\operatorname{Im} a_{A D}-\frac{1}{2}\left|a_{A D}\right|^{2} \operatorname{Im} r_{s, A D} k^{2}\right)}{k\left|1+i a_{A D} k-\frac{1}{2} a_{A D} r_{s, A D} k^{2}\right|^{2}} .
$$

Our truncation of the effective range expansion in Eq. (4) limits the validity of Eq. (11) to collision energies $E \ll$ $E_{D}$, where $E_{D}=\hbar^{2} /\left(m a^{2}\right)$. This expression could be extended straightforwardly to the entire region $E<E_{D}$ by using a parameterization of the atom-dimer phase shift below the dimer-breakup threshold given in Ref. [5]. The rate constant $\beta$ for $T \ll E_{D}$ can be obtained by inserting the cross section in Eq. (11) into Eq. (8). In the low-temperature limit, $\beta$ reduces to $6 \pi \hbar\left(-\operatorname{Im} a_{A D}\right) / m$, which can be expressed as [10]

$$
\beta=\frac{20.3 \sinh \left(2 \eta_{*}\right)}{\sin ^{2}\left[s_{0} \ln \left(a / a_{*}\right)\right]+\sinh ^{2} \eta_{*}} \frac{\hbar a}{m} .
$$

If $\eta_{*}$ is small, the maximum value of $\beta$ occurs when $a \approx$ $\left[1+\sinh ^{2} \eta_{*} /\left(2 s_{0}^{2}\right)\right] a_{*}$, which is $a \approx 1.0018 a_{*}$ if $\eta_{*}=0.06$.

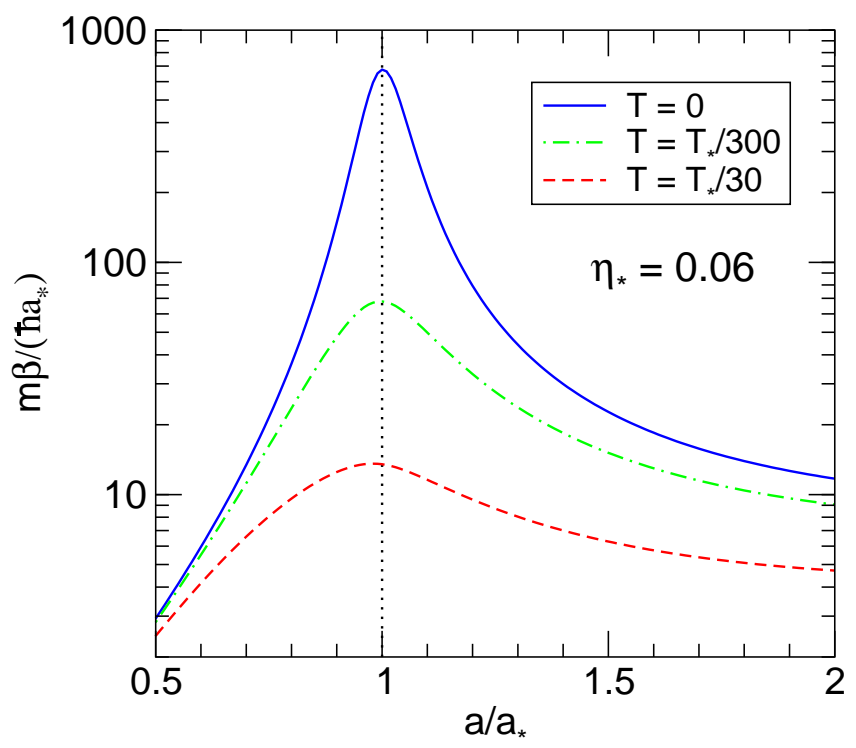

FIG. 1: The dimer relaxation length $m \beta / \hbar$ (in units of $a_{*}$ ) as a function of $a / a_{*}$ for $\eta_{*}=0.06$. The curves are for several values of the temperature: $T=0$ (solid line), $T_{*} / 300$ (dotdashed line), $T_{*} / 30$ (dashed line), where $k_{B} T_{*}=\hbar^{2} /\left(m a_{*}^{2}\right)$.

The resonant line shape for the dimer relaxation rate $\beta$ as a function of $a$ is illustrated in Figs. 1 and 2, In Fig. 1, we show the dimer relaxation length $m \beta / \hbar$ as a function of $a / a_{*}$ for $\eta_{*}=0.06$ and several values of $T$. The temperature is given in units of $T_{*}=\hbar^{2} /\left(k_{B} m a_{*}^{2}\right)$, which is the dimer binding energy for $a=a_{*}$. These results can be applied to any atomic system with large positive $a$ by using the appropriate value of $a_{*}$ to convert to physical units. In Fig. 2, we show $m \beta / \hbar$ as a function of $a / a_{*}$ for $T=T_{*} / 30$ and several values of $\eta_{*}$. As $T$ increases, the height of the resonant peak decreases and its width increases, but the location of the peak remains very close to $a_{*}$. If $\eta_{*}$ is increased, the height of the resonance peak and its width both increase. The discrete scaling symmetry implies that $\beta$ has similar peaks at $a \approx(22.7)^{n} a_{*}$, $n=1,2, \ldots$ with maximum values of $\beta$ that are larger by $(22.7)^{n}$. The fact that the peak remains near $a_{*}$ implies that $a_{*}$ (and therefore the Efimov parameter $\kappa_{*}$ ) can be determined accurately by measurements at nonzero temperature without any need for thermal corrections. The value of $\eta_{*}$ can be determined from the line shape of $\beta$ as 
a function of $a$, if the temperature is known to sufficient accuracy.

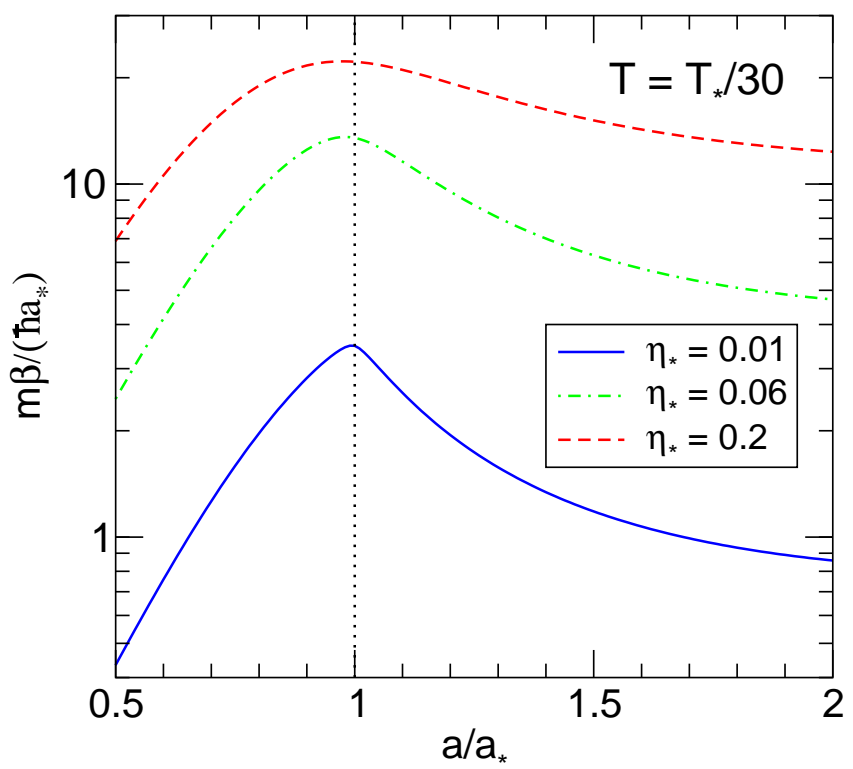

FIG. 2: The dimer relaxation length $m \beta / \hbar$ (in units of $a_{*}$ ) as a function of $a / a_{*}$ for $T=T_{*} / 30$, where $k_{B} T_{*}=\hbar^{2} /\left(m a_{*}^{2}\right)$. The curves are for $\eta_{*}=0.01$ (solid line), 0.06 (dot-dashed line), and 0.2 (dashed line).

The resonance in inelastic atom-dimer scattering observed by the Innsbruck group occurs when the scattering length is $a \approx 400 a_{0}$ [15]. Since the van der Waals length scale for Cs atoms is $\left(m C_{6} / \hbar^{2}\right)^{1 / 4} \approx 200 a_{0}$, this value of $a$ is not deep into the universal region. If we ignore this problem, we can apply our universal results to ${ }^{133} \mathrm{Cs}$ atoms in the vicinity of the resonance by setting $a_{*}=400 a_{0}$. Using $\hbar^{2} /\left(k_{B} m\right)=1.3032 \mathrm{~K} a_{0}^{2}$ for Cs atoms, we find that the temperature scale set by $a_{*}$ is $T_{*}=8145 \mathrm{nK}$. The temperatures in Fig. 1 correspond to $0,27.2 \mathrm{nK}$, and $272 \mathrm{nK}$. The temperature in Fig. 2 corresponds to $272 \mathrm{nK}$. The measurements of $\beta$ by the Innsbruck group were carried out at temperatures near $250 \mathrm{nK}$ [15]. A reasonable guess for $\eta_{*}$ is the value 0.06 that was measured for large negative $a$ on the other side of the zero of $a$. The value for $\eta_{*}$ for this region of large positive $a$ could be measured by fitting the line shape to our universal results, provided the temperature can be determined with sufficient accuracy.

Universality requires that Efimov physics be described by the same parameters for large positive and negative $a$ on opposite sides of a point where $a \rightarrow \pm \infty$. The ${ }^{133} \mathrm{Cs}$ experiment of Ref. 11] studied Efimov physics for large positive and negative $a$ on opposite sides of the zero that comes from the interplay between a broad Feshbach resonance near 0 magnetic field and the large off-resonant scattering length for ${ }^{133} \mathrm{Cs}$. Is there any relation between the Efimov parameters for the two regions on opposite sides of a zero of the scattering length created by a Fes- hbach resonance? Is there any relation between the Efimov parameters associated with the large off-resonant scattering length and the Efimov parameters associated with the various Feshbach resonances? These questions can be answered by accurate measurements of the Efimov parameters.

The Innsbruck experiment demonstrated that the resonant inelastic losses associated with an Efimov trimer at the 3 -atom threshold could be used to determine the Efimov parameters accurately for large negative $a$ [11]. The Innsbruck group has also observed resonant inelastic losses associated with an Efimov trimer at the atomdimer threshold [15]. The results we have presented should make it possible to use those results to determine the Efimov parameters accurately for large positive $a$. These parameters could then be used to make universal predictions for other phenomena associated with Efimov physics, such as the locations of local minima and maxima in the 3-body recombination rate.

We thank R. Grimm for valuable discussions. This research was supported in part by the Department of Energy under grant DE-FG02-05ER15715.

[1] V. Efimov, Phys. Lett. 33B, 563 (1970).

[2] V. N. Efimov, Sov. J. Nucl. Phys. 12, 589 (1971).

[3] V. N. Efimov, Sov. J. Nucl. Phys. 29, 546 (1979).

[4] E. Braaten and H.-W. Hammer, Phys. Rept. 428, 259 (2006) arXiv:cond-mat/0410417.

[5] E. Braaten and H.-W. Hammer, Phys. Rev. A 67, 042706 (2003) arXiv:cond-mat/0203421.

[6] E. Nielsen and J.H. Macek, Phys. Rev. Lett. 83, 1566 (1999).

[7] B.D. Esry, C.H. Greene, and J.P. Burke, Phys. Rev. Lett. 83, 1751 (1999).

[8] P.F. Bedaque, E. Braaten, and H.-W. Hammer, Phys. Rev. Lett. 85, 908 (2000) arXiv:cond-mat/0002365.

[9] E. Braaten and H.-W. Hammer, Phys. Rev. Lett. 87, 160407 (2001) arXiv:cond-mat/0103331.

[10] E. Braaten and H.-W. Hammer, Phys. Rev. A 70, 042706 (2004) arXiv:cond-mat/0303249.

[11] T. Kraemer, M. Mark, P. Waldburger, J.G. Danzl, C. Chin, B. Engeser, A.D. Lange, K. Pilch, A. Jaakkola, H.-C. Nägerl, and R. Grimm, Nature 440, 315 (2006).

[12] J.P. D'Incao, H. Suno, and B.D. Esry, Phys. Rev. Lett. 93, 123201 (2004).

[13] S. Jonsell, Europhys. Lett. 76, 8 (2006).

[14] M.T. Yamashita, T. Frederico, and L. Tomio, to appear in Phys. Lett. A (2007) arXiv:cond-mat/0608542.

[15] R. Grimm, talk at 18th International Few-Body Conference, Santos, Brazil, August 2006.

[16] I.V. Simenog and A.I. Sitnichenko, Doklady Academy of Sciences of the Ukrainian SSR, Ser. A, 11, 74 (1981).

[17] P.F. Bedaque, H.-W. Hammer, and U. van Kolck, Phys. Rev. Lett. 82, 463 (1999) arXiv:nucl-th/9809025.

[18] P.F. Bedaque, H.-W. Hammer, and U. van Kolck, Nucl. Phys. A 646, 444 (1999) arXiv:nucl-th/9811046. 